This article has undergone peer review, been accepted for publication, and posted online before the distribution of the complete issue. It has not yet undergone copy editing and may contain statements and information that have errors. The final version will be available upon completion. Visit www.springerpub.com for more info.

Journal of Cognitive Psychotherapy: An International Quarterly

Volume 35, Number 3, 2021

\title{
Brooding Over the Closet: Differentiating Sexual Orientation Rumination and Sexual Orientation Obsessions
}

\author{
Alexandria M. Luxon, MA \\ Illinois Institute of Technology, Chicago, IL \\ Gregory S. Chasson, PhD \\ Illinois Institute of Technology, Chicago, IL \\ Monnica T. Williams, PhD \\ University of Connecticut, Storrs, CT \\ Matthew D. Skinta, PhD \\ Roosevelt University, Chicago, IL \\ M. Paz Galupo, PhD \\ Towson University, Towson, $M D$
}

\begin{abstract}
Two concepts that describe repetitive thoughts regarding an individual's sexual orientationsexual orientation rumination and sexual orientation obsessions-have been introduced into the research literature. Despite the fact that these concepts have similarities, important distinctions exist with regard to their theoretical underpinnings, development, and catalyst of stress. As these concepts have never been teased apart in the research literature, understanding how these concepts are similar and different is particularly important. To this end, the present overview synthesizes the current literature regarding these concepts with the purpose of providing a decisional framework for differentiating sexual orientation rumination and sexual orientation obsessions and suggesting areas of future research.
\end{abstract}

Keywords: sexual orientation; sexual minority; minority stress; rumination; obsessions

ก wo concepts independently introduced in the current research literature describe the manifestation of repetitive thoughts about sexual orientation: sexual orientation rumination (Galupo \& Bauerband, 2016) and sexual orientation obsessions (Williams, 2008; Williams \& Farris, 2011). Sexual orientation rumination is defined as analyzing how one's sexual orientation may both negatively (rumination) and positively (reflection) impact one's life, including interactions with other people (Galupo \& Bauerband, 2016). Sexual orientation obsessions, previously referred to as homosexuality anxiety, is a specific type of sexual obsession that may occur within obsessive-compulsive disorder (OCD) (Williams, 2008). Williams (2008) indicates that 
This article has undergone peer review, been accepted for publication, and posted online before the distribution of the complete issue. It has not yet undergone copy editing and may contain statements and information that have errors. The final version will be available upon completion. Visit www.springerpub.com for more info.

168 Luxon et al.

sexual orientation obsessions include (a) persistent anxiety that one's heterosexual orientation will transition to a nonheterosexual orientation, (b) undesirable and invasive thoughts associated with sexual minority sexual behaviors, and/or (c) anxiety that other people will think one identifies as lesbian, gay, bisexual individuals, individuals questioning their sexual orientation, and other sexual minority individuals (i.e., LGBQ+). It should be noted that we intentionally use LGBQ+ across this article in order to avoid conflation of sexual orientation and gender identity, and to highlight that both sexual orientation rumination and sexual orientation obsessions are specifically conceptualized with regard to the sexual orientation identity and not gender identity.

Sexual orientation rumination and sexual orientation obsessions are similar in that they both describe the phenomena of repetitive and often distressing thoughts about sexual orientation. The two concepts, however, are distinct and influenced by differences between an individual's sexual orientation identity, attraction toward other people, and sexual behavior. Differentiating sexual orientation rumination from sexual orientation obsessions early in the process of case conceptualization is essential for providing effective mental health treatment.

Understanding the similarities and differences between sexual orientation rumination and sexual orientation obsessions first requires some context around sexual orientation. Sexual orientation is conceptualized as an internal mechanism that directs both sexual and romantic interests (Diamond, 2003; Rosario \& Schrimshaw, 2014). As a multi-dimensional concept, sexual orientation encompasses three primary components-identity, attraction, and behavior-that do not always predictably align (Bailey et al., 2016; Bauer \& Brennan, 2013; Laumann et al., 1994; Wolff et al., 2017). For example, a man can identify as heterosexual and be sexually involved both with women and men (Morgan et al., 2010; Ward, 2008). Another person may identify as bisexual, not base their attraction on gender, and only be sexually active within a monogamous relationship (Galupo et al., 2017). Research on sexuality is complicated because sexual attraction is most often conceptualized as continuous, while sexual behavior and sexual identity are conceptualized as categorical in nature (Pega et al., 2013). The continuous/categorical distinction may partially account for why these three components are not always congruent (Galupo, 2018). Research is further complicated when accounting for the way many individuals (including those who identify as heterosexual) question their sexual orientation at some point in their lives (Morgan \& Thompson, 2011; Morgan et al., 2010), experience sexual fluidity (i.e., changes in sexual attractions, desires, or behaviors within different contexts; Diamond, 2009; Katz-Wise, 2015), and/or use multiple identity labels across time and context (Galupo et al., 2015).

Perspectives in the field on sexual orientation identity and attraction have shifted progressively over the decades, resulting in less pathologizing of sexual minority individual's experiences; nonetheless, individuals may still legitimately present to treatment with concerns about their sexual identity. In the following sections, we review the literature framing sexual orientation rumination and sexual orientation obsessions, consider their similarities and differences, and propose a preliminary decisional framework to reconcile these two conceptualizations.

\section{Rumination About Sexual Orientation}

\section{Sexual Orientation Rumination Versus Depressive Rumination}

The majority of past research focusing on sexual minority stress and rumination has utilized measures of depressive rumination. Depressive rumination can include searching for causes of depression, focusing on problems that others do not experience, or repetitively dwelling on past situations (Trapnell \& Campbell, 1999; Treynor et al., 2003). Previous research has found that sexual minority individuals consistently demonstrated higher rates of depressive rumination compared to heterosexual individuals (Hatzenbuehler et al., 2008). Furthermore, Borders et al. (2014) found 
This article has undergone peer review, been accepted for publication, and posted online before the distribution of the complete issue. It has not yet undergone copy editing and may contain statements and information that have errors. The final version will be available upon completion. Visit www.springerpub.com for more info.

that sexual minority individuals' uncertainty about their sexual orientation resulted in higher rates of depressive rumination, while this association was not found for heterosexual individuals. Hatzenbuehler (2009) theorized that stress related to sexual minority stigma likely increases one's chances of experiencing depressive rumination. Furthermore, identity concealment increases an individual's risk for other issues including preoccupation (e.g., sexual minority individuals' constant fear of encountering stigma or discovery of their identity), constant questioning (e.g., sexual minority individuals questioning which situations to conceal or reveal their identity), and self-monitoring (i.e., changing how one behaves or interacts with others so as not to reveal their sexual minority identity; Hatzenbuehler, 2009).

While research has demonstrated that sexual minority individuals experience higher rates of depressive rumination compared to heterosexual individuals, Galupo and Bauerband (2016) also found that sexual minority individuals experience identity-specific rumination centering on repetitive thinking about their sexual orientation (i.e., sexual orientation rumination). In order to characterize this type of rumination, Galupo and Bauerband (2016) developed a scale to measure repetitive thinking about one's sexual orientation identity; this measure allows sexual orientation rumination to be disaggregated from depressive rumination. The Sexual Orientation Rumination and Reflection Scale (SRRS) includes four factors: reflection, rumination, preoccupation with others' perceptions, and perseveration. The reflection and rumination subscales of the SRRS parallel the way depressive rumination scales (Trapnell \& Campbell, 1999; Treynor et al., 2003) and other identity rumination measures (Bauerband \& Galupo, 2014) include both positive and negative thoughts. Reflection includes contemplating one's sexual orientation identity in positive ways (e.g., "look at my sexual orientation identity in philosophical ways"), while rumination is characterized as a focus on one's negative thoughts about their sexual orientation (e.g., "think that life would be easier if I were heterosexual"). As a scale specific to rumination about sexual orientation, however, the SRRS captures two unique aspects related to thinking about sexual orientation identity. Preoccupation with others' perceptions is characterized by excessive focus on how other people judge or view the individual based on the individual's sexual orientation (e.g., "Try to figure out what others think about my sexual orientation") and perseveration includes recurring thoughts related to one's sexual orientation identity (e.g., "I can't stop thinking about my sexual orientation").

Despite the positive components of reflection, Galupo and Bauerband (2016) found that all components of sexual orientation rumination were associated with negative identity (e.g., homonegativity, an individual's need for privacy, necessity for acceptance, and difficulties processing identity). Internalized homophobia (i.e., negative thoughts and experiences one may have as a result of their minority identity), identity salience (i.e., the importance of one's sexual orientation in their life), and overall sexual minority identity explained nearly half of their sample's SRRS scores, but only explained 10\%-23\% of depressive rumination scores (Galupo \& Bauerband, 2016). This result suggests that sexual orientation rumination is a distinct concept from depressive rumination. Furthermore, within-group differences on rumination among sexual minority individuals who identify differently (e.g., lesbian vs. bisexual) has emerged, highlighting the centrality of sexual orientation identity to sexual orientation rumination (Galupo \& Bauerband, 2016; Galupo et al., 2015).

Due to the way sexuality is conceptually normalized on heterosexist assumptions (Galupo, Davis, et al., 2014; Galupo et al., 2017; van Anders, 2015), it is expected that most individuals who identify as a sexual minority experience some degree of sexual orientation rumination at some point in their lives. Indeed, consistent with the notion that individuals in a heterosexist society are raised with a presumptive heterosexual identity that is challenged by the awareness of attraction to the same sex, most theories of sexual orientation development begin with a period of uncertainty, termed identity confusion. In this initial phase, individuals question their previously held 
This article has undergone peer review, been accepted for publication, and posted online before the distribution of the complete issue. It has not yet undergone copy editing and may contain statements and information that have errors. The final version will be available upon completion. Visit www.springerpub.com for more info.

170 Luxon et al.

beliefs and view of their heterosexual identity (McCarn \& Fassinger, 1996). Thus, it is important to note that although some level of sexual orientation rumination may be expected, not all experiences of sexual orientation rumination are associated with clinically significant stress or require clinical intervention.

\section{Minority Stress Model Underlying Sexual Orientation Rumination}

Sexual orientation rumination is conceptualized from the minority stress model, which posits that sexual minority individuals experience both distal and proximal forms of stress (Meyer, 2003, 2013). Distal experiences of stress are life experiences, such as violence or harassment, which occur due to an individual's sexual orientation (Meyer, 2003, 2013). Higher rates of victimization and harassment have been well established for both sexual minority youth (Friedman et al., 2011) and adults (Katz-Wise \& Hyde, 2012), and these distal stressors often reoccur throughout a sexual minority person's life (Meyer, 2003, 2013). Proximal stressors are internal thought processes that occur as a reaction to both distal stressors and societal expressions of heterosexism (Meyer, 2003, 2013). Examples of common forms of proximal stressors include hypervigilence, expectation of rejection, concealment of identity, and internalization of negative societal opinions (i.e., internalized homophobia/heterosexism; Meyer, 2003, 2013). Previous research indicates that as sexual minority individuals experience increased rates of hypervigilance, expectation of rejection, concealment of identity, and internalized homophobia/heterosexism, they are at increased risk of experiencing rumination in an attempt to cope with sexual minority stress (Hatzenbuehler, 2009).

\section{Obsessions About Sexual Orientation}

Another way individuals could be experiencing repetitive thoughts about sexual orientation is called sexual orientation obsessions, which are often but not always manifested as part of a larger condition, OCD (Wetterneck et al., 2011; Williams, 2008; Williams \& Farris, 2011). Affecting $2.3 \%$ of people in the United States in their lifetime (Ruscio et al., 2010), OCD is characterized by obsessions, which refer to recurrent intrusive thoughts, images, or impulses that cause distress, which in turn elicit compulsions, which are repetitive behaviors or mental actions that function to decrease obsessional distress (American Psychiatric Association, 2013). Prominent motifs of obsessions and compulsions vary extensively, with common themes including cleanliness, symmetry, order, and forbidden or taboo thoughts (Williams et al., 2013). The latter category, taboo thoughts (e.g., sexual thoughts, including sexual orientation intrusions), is the focus of the current discussion.

While a case study, clinical observations, and recent findings also suggest that it may be possible for sexual minority individuals to have sexual orientation obsessions about one's orientation changing to a heterosexual orientation, the emphasis here is on the more prevalent presentation of the heterosexual experience of sexual orientation obsessions (Goldberg, 1984; Williams et al., 2018). Individuals with sexual orientation obsessions often doubt and question the significance and meaning of their thoughts and come to interpret these thoughts as indicating they are a sexual minority (Williams, 2008). They also worry about the consequences these thoughts will have on their life, such as no longer being able to have relationships with individuals of their preferred sex (e.g., individuals of a different sex for heterosexual individuals; Williams, 2008). Sexual orientation obsessions are associated with increased distress, interference, and avoidance, compared to other presentations of OCD symptoms (Williams \& Farris, 2011). In one study, 21\% of individuals experiencing sexual orientation obsessions were so distressed that they reported thoughts of suicide (Williams et al., 2014). This suggests that individuals with sexual orientation obsessions may represent a high-risk subgroup with potential public health implications. 


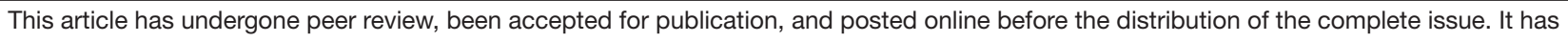
not yet undergone copy editing and may contain statements and information that have errors. The final version will be available upon completion. Visit www.springerpub.com for more info.

Thus, nested within a conceptual hierarchy, sexual orientation obsessions are a manifestation of sexual obsessions (Williams, 2008), which in turn is a type of taboo thought (Pinto et al., 2007), which is a symptom dimension often found in OCD (Williams et al., 2013). Although not an epidemiological study, Williams and Farris (2011) found that among individuals who were seeking treatment from a variety of OCD clinics, $8 \%$ reported current sexual orientation obsessions and $11.9 \%$ endorsed lifetime symptoms. However, individuals not diagnosed with OCD may also experience sexual obsessions (Wetterneck et al., 2011), which may include sexual orientation obsessions.

\section{Cognitive and Behavioral Theories of Obsessions}

Obsessions are primarily conceptualized from a cognitive theoretical model. Rachman (1997) proposed that obsessions occur because people misinterpret the importance of experiences that are external (e.g., individuals experience flirtation from a person of the same sex and worry this means they are LGBQ+) or internal (e.g., people have a thought that another individual of the same sex is attractive, signaling they are LGBQ+). Among individuals with obsessional tendencies, distress from these misinterpretations is compounded by a tendency to believe that having a thought is the same as engaging in the behavior (e.g., experiencing an intrusive thought of groping someone of the same sex is equivalent to actually groping them). Distress also stems from belief that such thoughts require personal control, and if individuals fall short of that control, they are to blame (Salkovskis, 1985). Individuals often assume the worst possible outcome associated with obsessions, and these interpretations result in fear and anxiety (Rachman, 1997). This fear and anxiety may be further fueled by components of social cognitive theory (Bandura, 1986), which emphasizes observational learning within a social context. For individuals who are distressed about experiencing sexual orientation obsessions or who feel stigmatized for these thoughts, they may be more hypervigilant to situations and others' behaviors that reinforce negative views of LGBQ+ individuals.

The cognitive conceptualization of obsessions is combined with a longstanding behavioral theoretical model of anxiety. Mowrer (1947) proposed a two-factor model, with the second factor highlighting the maintenance of ritualistic behavior to reduce the distress caused by obsession. Mowrer's model suggests that compulsive behaviors are performed as a strategy to reduce distress stemming from obsessions, thereby negatively reinforcing the compulsive behavior. As an example with sexual orientation obsessions, an individual may engage in ritualistic behavior, such as excessively viewing same-sex pornography, as a way of testing arousal to same-sex stimuli (e.g., Williams et al., 2011). Lack of arousal would reassure the individual that they are not LGBQ+, reducing distress from the sexual orientation obsession. The ritualistic testing behavior of reviewing same-sex pornography is therefore negatively reinforced (i.e., reduction of a negative experience-anxiety-from the obsession) and increases the likelihood that such testing rituals would happen again in the future as a strategy for dealing with this type of distress. This is the cognitive-behavioral cycle that characterizes the nature of sexual orientation obsessions.

\section{Why Differentiate Sexual Orientation Rumination and Sexual Orientation Obsessions?}

If sexual orientation rumination and obsessions are not differentiated, mental health clinicians could make incorrect assumptions about an individual's sexual orientation. Such assumptions may also be associated with other issues such as over-pathologizing individuals who need help affirming their sexual orientation, improperly diagnosing individuals and thus administering improper treatments, and/or increasing an individual's experience of stigma and distress (Glazier et 
al., 2013; Williams et al., 2014). If any of these were to occur in a treatment setting, clients might prematurely terminate therapy before receiving assistance to decrease their distress, or could actually be harmed by therapy. In addition, treatment for sexual identity rumination versus sexual orientation obsessions may be fundamentally different. This issue is complicated, as no treatment studies have been conducted with sexual orientation rumination specifically to determine the optimal treatment. Regardless of one's sexual orientation, the American Psychological Association (APA) recommends against any attempts to change one's sexual orientation as these attempts could be considered unethical with research suggesting the potential for it to cause harm to the individual; instead it is recommended for sexual minority individuals that one should treat the symptoms a client experiences that are associated with minority stress (American Psychological Association [APA], 2012; American Psychological Association, Task Force on the Appropriate Therapeutic Responses to Sexual Orientation, 2009; Harper et al., 2013). Initial research efforts on therapy for alleviating minority stress and its associated issues more broadly are reviewed elsewhere, but have only been tested on gay and bisexual men (e.g., Burton et al., 2017).

\section{Differences Between Sexual Orientation Rumination and Sexual Orientation Obsessions}

The primary differences between sexual orientation rumination and sexual orientation obsessions are summarized in Figure 1. The first level of this figure represents differences in the psychosocial theoretical frameworks from which sexual orientation rumination and sexual orientation obsessions are conceptualized. Phenomenological differences between sexual orientation rumination and obsessions are presented in the lower two levels of the figure and are likely to emerge as part of the direct presentation of individuals experiencing sexual orientation rumination or sexual orientation obsessions. These phenomenological differences may be the starting point for differentiating sexual orientation obsessions from distressing sexual orientation rumination in a clinical setting.

The theoretical frameworks accounting for sexual orientation rumination and obsessions are distinct. As outlined above, the predominate theoretical model for sexual orientation rumination is that of the minority stress model (Meyer 2003, 2013), whereas the primary theoretical

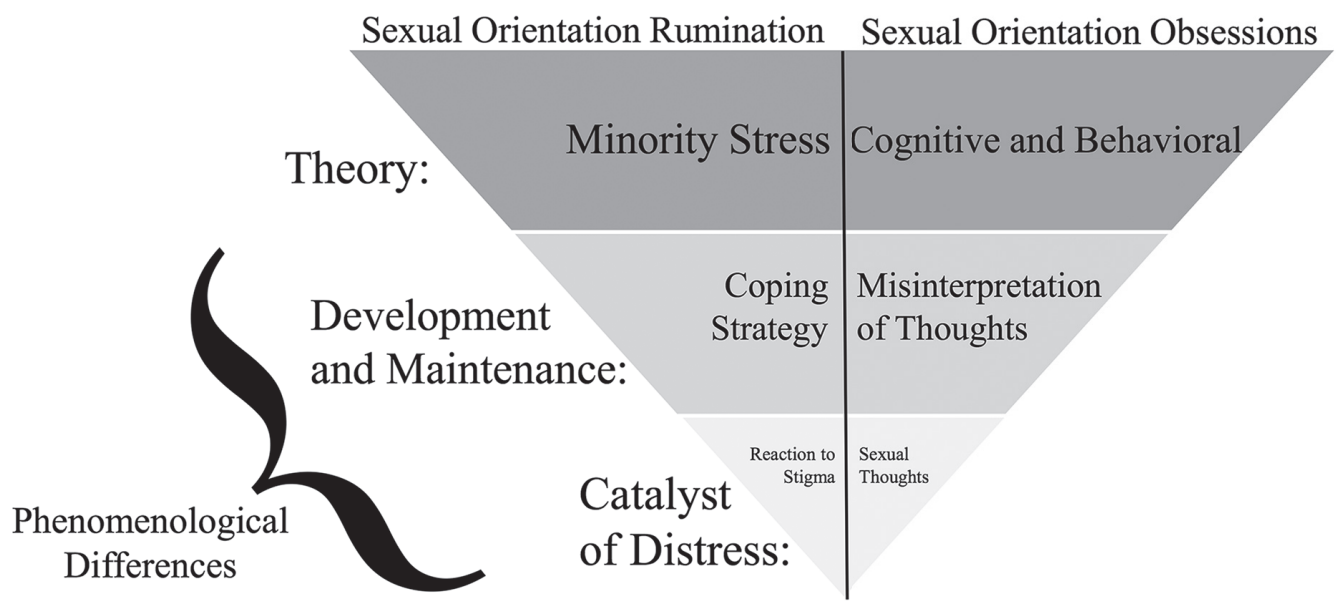

FigURE 1. Primary differences between sexual orientation rumination and sexual orientation obsessions. 
This article has undergone peer review, been accepted for publication, and posted online before the distribution of the complete issue. It has not yet undergone copy editing and may contain statements and information that have errors. The final version will be available upon completion. Visit www.springerpub.com for more info.

models for sexual orientation obsessions are cognitive and behavioral theories (Rachman, 1997; Salkovskis, 1985) and negative reinforcement of compulsive behavior (Mowrer, 1947). This is not to say that sexual orientation rumination and obsessions are dissimilar in all theoretical underpinnings. For example, Bandura's (1986) social cognitive theory on observational learning likely accounts for distress both in sexual orientation rumination and sexual orientation obsessions, because individuals experiencing either may internalize the stigma of having a sexual minority identity through observational learning

Based on the second level of Figure 1, sexual orientation rumination and sexual obsessions appear to differ based on how each develop and their maintenance. In part, sexual orientation rumination is a reaction to external and internal stigma associated with minority identity and therefore appears to develop as a coping mechanism in response to this stigma (e.g., Galupo \& Bauerband, 2016; Hatzenbuehler, 2009; Meyer, 2003, 2013). This is supported by research suggesting that sexual orientation rumination is responsive to societal events that are perceived as relevant to the rights and protection of individuals in the LGBQ+ community. For example, Gonzalez et al. (2018) found that sexual minority individuals reported increased sexual orientation rumination after the 2016 Presidential election, a time when LGBQ+ rights were disputed and widely discussed in the media. It is well established that an adversarial political climate surrounding the LGBQ+ community can create opportunities for resilience or distress (e.g., Russell \& Richards, 2003); similar to the coping mechanism of sexual orientation rumination possibility leading to either resilience or distress (Galupo \& Bauerband, 2016; Hatzenbuehler, 2009). On the other hand, because research suggests that most individuals experiencing sexual orientation obsessions are predominately heterosexual and not LGBQ+ (Williams et al., 2018), these obsessions appear to develop less as a coping mechanism or reaction to stigma and more as a result of overestimation of negative outcomes (Rachman, 1997) and misinterpretation of one's thoughts (Salkovskis, 1985).

Lastly, regarding the last level of Figure 1, if individuals experiencing sexual orientation rumination or sexual orientation obsessions experience associated stress, the origin of that stress appears to differ based on consistency with other aspects of one's sexual orientation, including patterns of social and emotional connection, fantasy, and arousal. Individuals experiencing sexual orientation rumination are typically not stressed by an inconsistency in their sexual orientation identity, same-sex attractions, or same-sex behaviors. Indeed, the sexual orientation thoughts tend to be consistent with the same-sex thoughts, attractions, or arousals they are experiencing. However, when presenting to a clinical setting, they are often stressed by the sexual minority stigma, harassment, discrimination, and/or violence they experience due to their identity (Galupo \& Bauerband, 2016). On the other hand, individuals experiencing sexual orientation obsessions are stressed by the inconsistency between their sexual orientation identity and the same-sex thoughts, attractions, or arousals they are experiencing (Williams, 2008). The obsessions, by nature, tend to be inconsistent with an individual's historical patterns of sexual thoughts, attractions, and arousal. However, clinicians must also be careful not to rely too heavily on this distinction, as initial evidence has suggested that individuals struggling with sexual orientation obsessions may eventually identify as LGBQ+ despite initial distress, or experience sexual orientation obsessions and sexual orientation rumination concurrently (Williams et al., 2018).

It remains unclear if sexual arousal patterns differ between sexual orientation rumination and sexual orientation obsessions. Sexual orientation rumination is focused exclusively on one's identity, not on arousal (Galupo \& Bauerband, 2016). Furthermore, individuals who experience sexual orientation obsessions may experience arousal for same or different-sex individuals (Williams \& Wetterneck, 2019). Thus, sexual arousal may not be a reliable and valid method to differentiate sexual orientation rumination from sexual orientation obsessions. Ultimately, however, the research on sexual arousal vis-à-vis these two types of repetitive sexual thoughts is limited. Future research is needed to suss out any possible differences in arousal. 


\section{Similarities Between Sexual Orientation Rumination AND OBSESSIONS}

Although sexual orientation rumination and sexual orientation obsessions emerged from two different theoretical frameworks (see Figure 1, discussed previously), they have important similarities. While different terminology may be adopted to characterize the nature of obsessive and ruminative thoughts, the concepts are similar, particularly in their repetitive, intrusive, and interfering nature (Wahl et al., 2011). In addition to both being repetitive, intrusive, and interfering, sexual orientation rumination and obsessions are also similar in some themes of thought content. These similar themes can be gleaned from two self-report instruments that have been constructed to measure sexual orientation rumination (i.e., SRRS; Galupo \& Bauerband, 2016) and sexual orientation obsessions (Sexual Orientation Obsessions and Reactions Test [SORT]; Williams et al., 2018). One shared theme between sexual orientation rumination and sexual orientation obsessions is that both involve excessive thoughts about changes or stability of one's sexual orientation. For instance, individuals experiencing sexual orientation rumination may reassess if they still identify as LGBQ+ (Galupo \& Bauerband, 2016), and individuals experiencing obsessions fear that their sexual orientation is changing from a desired to an undesired identity (Williams, 2008; Williams et al., 2018).

A second factor that must be considered for individuals experiencing sexual orientation rumination or obsessions is the experience of stigma. It seems likely that individuals experiencing either phenomenon are negatively impacted by LGBQ+ stigma. Previous research has indicated that sexual orientation rumination is predominantly associated with experiencing stigma related to sexual minority identity (Hatzenbeuhler, 2009). Furthermore, because individuals with sexual orientation obsessions fear they will become LGBQ+, it is possible that they also experience $L G B Q+$ stigma, similar to LGBQ+ individuals. This possibility needs further research. Unfortunately, individuals experiencing sexual orientation obsessions or sexual orientation rumination may also experience stigma associated with mental health symptoms. On the one hand, individuals with sexual orientation obsessions and a diagnosis of OCD may experience stigma associated with their OCD symptoms (Chasson et al., 2018). For example, previous research has found that sexual obsessions, of which sexual orientation obsessions is a symptom dimension, are stigmatized more by clinicians and clinicians in training than scrupulosity obsessions (Steinberg \& Wetterneck, 2017). Furthermore, participants who are not in the mental health field stigmatized individuals with sexual obsessions more than those with contamination obsessions (Cathey \& Wetterneck, 2013). On the other hand, research has indicated that LGBQ+ individuals, in response to minority stigma, experience a higher level of mental health symptoms (Meyer, 2003, 2013; Plöderl \& Tremblay, 2015), which may result in them also experiencing stigma associated with psychiatric conditions, but not necessarily an OCD diagnosis. However, this is not true of all LGBQ+ individuals.

A final shared factor between sexual orientation rumination and obsessions is that individuals fear how others will treat them due to their sexuality or assumed sexuality. That is, individuals appear to be distressed about other people's reaction to their actual or assumed sexuality or their same-sex thoughts (Galupo \& Bauerband, 2016; Williams, 2008; Williams et al., 2018). As a result, individuals experiencing sexual orientation rumination or sexual orientation obsessions are likely to be attentive to their same-sex thoughts and desires. As another example of this shared theme, individuals experiencing sexual orientation rumination and sexual orientation obsessions contemplate how their lives have been or may be impacted by an LGBQ+ sexual orientation. For example, both constructs are associated with worry about harassment or prejudice due to being perceived as LGBQ+ (Galupo \& Bauerband, 2016; Williams, 2008; Williams et al., 2018). 
This article has undergone peer review, been accepted for publication, and posted online before the distribution of the complete issue. It has not yet undergone copy editing and may contain statements and information that have errors. The final version will be available upon completion. Visit www.springerpub.com for more info.

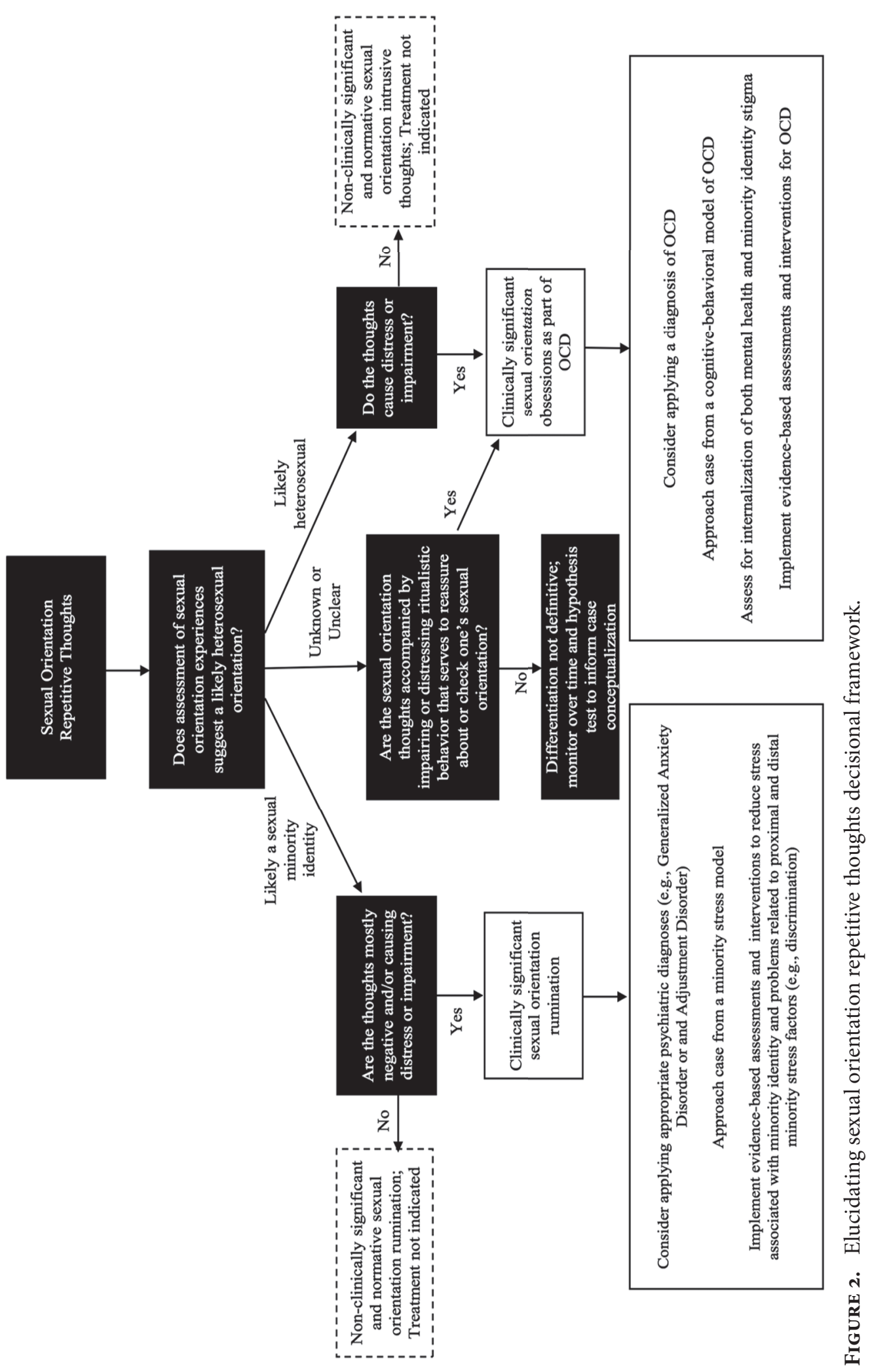


This article has undergone peer review, been accepted for publication, and posted online before the distribution of the complete issue. It has not yet undergone copy editing and may contain statements and information that have errors. The final version will be available upon completion. Visit www.springerpub.com for more info.

176 Luxon et al.

When summarizing the similarities in the themes of thought content between sexual orientation rumination and sexual orientation obsessions, it is apparent that both contain thoughts that appear to focus on current or future situations, such as how an individual would be treated for their same-sex thoughts and/or desires (Galupo \& Bauerband, 2016; Williams, 2008; Williams et al., 2018). This demonstrates a conceptual disaggregation between sexual orientation rumination and sexual orientation obsessions from typical depressive rumination, which primarily focuses on past experiences (Nolen-Hoeksema et al., 2008).

\section{Decisional Framework for Differentiating Sexual Orientation Repetitive Thoughts}

This article proposes a preliminary decisional framework for Elucidating Sexual Orientation Repetitive Thoughts (eSORT) as best reflective of either sexual orientation rumination or sexual orientation obsessions. Although based on empirical evidence, this decisional framework is preliminary in that additional research is needed for validation. Nonetheless, a decisional framework not only would facilitate accurate characterization of distressing repetitive sexual orientation thoughts for those who are seeking help from professionals or for those involved in research on these topics, but also help differentiate problematic, clinically significant sexual orientation thoughts from normal, nondistressing sexual orientation thoughts. eSORT is illustrated in Figure 2.

The first requirement in this framework is determining if the repetitive thoughts are consistent with historic aspects of one's sexual orientation. As discussed above, previous research indicates that individuals experiencing sexual orientation rumination view their LGBQ+ identity as being consistent with their repetitive LGBQ+ sexual orientation thoughts (Turner et al., 1992). On the other hand, previous research indicates that individuals beset with sexual orientation obsessions often view their sexual orientation-related thoughts as in conflict with their sexual orientation.

There are circumstances, however, when coherent and consistent self-awareness of one's sexual orientation is unknown or ambiguous. Indeed, as previously mentioned, most theories of sexual orientation development (which typically initiates in adolescence), begin with a period of identity confusion characterized by the individual questioning previously held beliefs, as well as their identity (McCarn \& Fassinger, 1996). Individuals who are questioning their sexual orientation may view LGBQ+-related thoughts as inconsistent with their sexual orientation, which was presumed to be heterosexual. In this sense, sexual orientation rumination that occurs during identity confusion, topographically, share characteristics of obsessions. Thus, it may be the case that most individuals who ultimately end up identifying as LGBQ+ pass through a phase or time of experiencing sexual orientation obsessions as they question their sexual orientation (i.e., during the identity confusion stage). For these individuals, they may experience sexual orientation obsessions without an OCD diagnosis. As a result, sexual orientation obsessions may initially appear dysfunctional but may serve as an initial response to questioning one's sexual orientation.

In addition, the prevalence of discrimination and violence toward sexual minority individuals in society may further encourage people who are questioning their sexual orientation to view their thoughts as a source of distress. One helpful clinical tool might be the Klein Sexual Orientation Grid (KSOG; Klein et al., 1985). Though the KSOG makes certain assumptions about cisgenderism and monosexual orientations, it also captures some of the nuanced experiences individuals have with differences of sexual identity, fantasy, emotional connections, and behavior across the past, present, and ideal future (e.g., Galupo et al., 2014; Galupo, Mitchell, et al., 2014). In clinical experience, it is not uncommon that an individual experiencing sexual orientation rumination or the possibly normative sexual orientation obsessions associated with self-realization 
This article has undergone peer review, been accepted for publication, and posted online before the distribution of the complete issue. It has not yet undergone copy editing and may contain statements and information that have errors. The final version will be available upon completion. Visit www.springerpub.com for more info.

might indicate an "ideal" orientation toward a different sex, with current and past fantasy, arousal, and emotional desires directed toward the same sex. This can be a helpful place to explore the individual's experiences in a compassionate way, and to affirm or normalize their fears of bias that may be encountered living openly as an LGBQ+-identified individual. In contrast, an individual experiencing sexual orientation obsessions characteristic of OCD are more likely to indicate past and present attraction, fantasy, and social or emotional connections to a different sex, though the question of an ideal future orientation may lead to distress, confusion, or uncertainty.

Based on eSORT, unknown or ambiguous self-identification would necessitate asking if the individual carries out compulsive behaviors related to sexual orientation repetitive thoughts. As described above, most forms of sexual orientation obsessions coincide with compulsions that aim to reassure or affirm one's sexual orientation (e.g., watching same-sex pornography to test arousal). On the other hand, individuals with sexual orientation rumination demonstrate preoccupation with how other people view them based on their sexual orientation (Galupo \& Bauerband, 2016), but they do not typically demonstrate compulsive behaviors to reaffirm or check sexual orientation.

An important point of eSORT is determining if the individual's sexual orientation repetitive thoughts cause distress and/or impairment. For individuals experiencing sexual orientation rumination, not only is it necessary to determine if there is distress or impairment, it is also necessary to determine if the individual is experiencing primarily negative thoughts about their sexual orientation, because rumination likely includes a mixture of negative thoughts with positive, nondistressing reflective thoughts about one's LGBQ+ identity. These individuals may require supportive therapy to relieve their distress, but they do not require a psychiatric diagnosis, as this could exacerbate stigma. However, if an individual's thoughts are mostly negative or they are experiencing distress or impairment due to the negative thoughts, they are likely experiencing clinically significant sexual orientation rumination. For these individuals, an appropriate psychiatric diagnosis - such as generalized anxiety disorder or an adjustment disorder-might be warranted. In addition, for such individuals, it would be important to assess the impact of distal and proximal stressors, particularly those related to the sexual minority stigma, harassment, discrimination, rejection, and/or violence related to their identity, attractions, and behaviors (Galupo \& Bauerband, 2016). In the event that someone presents with clinically significant sexual orientation rumination, it would be necessary to implement evidence-based assessment and interventions (e.g., Burton et al., 2017).

For individuals who are experiencing sexual orientation obsessions, it must also be determined if these thoughts are causing distress and/or impairment. For individuals not experiencing distress or impairment, they are likely experiencing nonclinically significant and normative sexual orientation intrusive thoughts. On the other hand, if an individual does experience distress and/ or impairment related to the thoughts, they likely are experiencing clinically significant sexual orientation obsessions and a diagnosis of OCD should be considered. Regardless of whether a diagnosis of OCD is applicable or not, a CBT framework and therapeutic approach, possibly including the use of exposure and response prevention, should be applied when treating sexual orientation obsessions (Williams et al., 2014). ${ }^{1}$

It is unclear if the model would be different for adults and youth. Sexual orientation obsessions or intrusive thoughts may be more common for youth because this is when people typically start questioning their sexual orientation (Calzo et al., 2011; Floyd \& Stein, 2002). Youth may therefore experience greater conflict between their sexual orientation identity and their attraction and sexual behaviors, resulting in greater experience of sexual orientation obsessions or intrusive thoughts compared to sexual orientation rumination. Ultimately, however, developmental differences in this model are unknown and represent a rich area for future research. 
This article has undergone peer review, been accepted for publication, and posted online before the distribution of the complete issue. It has not yet undergone copy editing and may contain statements and information that have errors. The final version will be available upon completion. Visit www.springerpub.com for more info.

178 Luxon et al.

\section{Clinical Applications}

When attempting to determine if an individual is experiencing sexual orientation rumination or obsessions, it is important to inquire if an individual can characterize their sexual orientation. However, it is important to remember that assessing sexual orientation is complicated and an individual may indicate identifying as heterosexual but may also be questioning their sexual orientation or think they are gay because of the obsessions they are experiencing. A follow-up question may consist of asking if the sexual orientation thoughts are causing distress or impairment. This question allows a clinician to determine if a diagnosis may or may not be warranted. It may be helpful to inquire if the individual is experiencing a mixture of negative and positive or reflective thoughts about their sexual orientation, because sexual orientation rumination often includes a positive or reflective component. This is not the case for sexual orientation obsessions. Furthermore, a clinician should assess if the client is engaging in compulsive-like behaviors related to their thoughts, as sexual orientation obsessions are often accompanied by sexual orientation compulsions, whereas sexual orientation rumination does not include such compulsive behaviors. Clinicians may also find it helpful to adopt the measure that assesses sexual orientation rumination called the SRRS (Galupo \& Bauerband, 2016). Two scales measure sexual orientation obsessions: the Sexual Orientation Obsessions and Reaction Test (Williams et al., 2018). These questions and questionnaires can help guide the clinician during case conceptualization and the treatment selection of an LGBQ+ affirmative therapy for sexual orientation rumination (e.g., Burton et al., 2017) or exposure and response prevention for obsessions, compulsions, and OCD more broadly (Williams et al., 2014).

\section{Conclusion}

The field of psychology has been fraught with controversy related to how sexual orientation has been discussed, assessed, treated, and researched. To aid in clarifying two forms of sexual orientation concerns that people may experience, we have developed a preliminary decisional framework called eSORT. Within eSORT, sexual orientation rumination and sexual orientation obsessions are differentiated by an individual's level of self-identification with their sexual orientation thoughts, the presence or absence of compulsive behaviors, and the level of distress/ impairment they experience related to sexual orientation repetitive thoughts. Based on the current research, individuals whose experience of sexual orientation attraction, arousal, and fantasy are consistent with their LGBQ+-related thoughts likely experience sexual orientation rumination as a result of stress related to the formation of a minority identity (Meyer, 2003, 2013). On the other hand, individuals whose experience of their heterosexual orientation is inconsistent with their LGBQ+-related thoughts likely experience sexual orientation obsessions. Furthermore, experiencing distress and/or impairment, regardless of whether an individual experiences sexual orientation rumination or sexual orientation obsessions, may necessitate a clinical diagnosis and the application of empirically based treatment in line with the specific diagnosis; however, a costbenefit analysis of diagnosis is necessary as diagnosis may potentially increase an individual's experience of stigma (i.e., stigma associated with psychiatric conditions). With additional research and validation, this preliminary framework has the potential to clarify these concepts for use in both clinical and research settings. Additional future research can inquire how both of these concepts are expressed in LGBQ+ and heterosexual individuals, to determine if they differ in degree between individuals of different sexual orientation or differ based on one's stage of sexual orientation identity development. Furthermore, research needs to investigate how these constructs play 
This article has undergone peer review, been accepted for publication, and posted online before the distribution of the complete issue. It has not yet undergone copy editing and may contain statements and information that have errors. The final version will be available upon completion. Visit www.springerpub.com for more info.

a role in the Meyer's $(2003,2013)$ minority stress model and Hatzenbuehler's (2009) psychological mediation framework especially the later which sites general rumination, but not sexual orientation rumination, as a key variable.

\section{Note}

1. Gender is not included as a variable in the decisional framework as gender of the individual is less informative than their self-identification, presence of compulsions, and level of distress and/or impairment. Furthermore, gender should not be used as a shortcut to determine if the individual is experiencing sexual orientation rumination or sexual orientation obsessions because this could lead to false assumptions.

\section{REFERENCES}

American Psychological Association. (2012). Guidelines for psychological practice with lesbian, gay, and bisexual clients. American Psychologist, 67, 10-42. https://doi.org/10.1037/a0024659

American Psychiatric Association. (2013). Diagnostic and statistical manual of mental disorders (5th ed.). American Psychiatric Publishing.

American Psychological Association, Task Force on the Appropriate Therapeutic Responses to Sexual Orientation. (2009). Report of the APA task force on the appropriate therapeutic reponses to sexual orientation. https://www.apa.org/pi/lgbt/resources/sexual-orientation

Bailey, J. M., Vasey, P. L., Diamond, L. M., Breedlove, S. M., Vilain, E., \& Epprecht, M. (2016). Sexual orientation, controversy, and science. Psychological Science in the Public Interest, 17, 45-101. https://doi. org/10.1177/1529100616637616

Bandura, A. (1986). Social foundations of thought and action: A social cognitive theory. Prentice-Hall.

Bauerband, L. A., \& Galupo, M. P. (2014). The gender identity reflection and rumination scale: Development and psychometric evaluation. Journal of Counseling \& Development, 92, 219-231. https://doi. org/10.1002/j.1556-6676.2014.00151.x

Bauer, G. R., \& Brennan, D. J. (2013). The problem with 'behavioral bisexuality': Assessing sexual orientation in survey research. Journal of Bisexuality, 13, 148-165. https://doi.org/10.1080/15299716.2013.782260

Borders, A., Guillén, L. A., \& Meyer, I. H. (2014). Rumination, sexual orientation uncertainty, and psychological distress in sexual minority university students. The Counseling Psychologist, 42, 497-523. https:// doi.org/10.1177/0011000014527002

Burton, C. L., Wang, K., \& Pachankis, J. E. (2017). Psychotherapy for the spectrum of sexual minority stress: Application and technique of the esteem treatment model. Cognitive and Behavioral Practice, 26(2), 285-299. https://doi.org/10.1016/j.cbpra.2017.05.001

Calzo, J. P., Antonucci, T. C., Mays, V. M., \& Cochran, S. D. (2011). Retrospective recall of sexual orientation identity development among gay, lesbian, and bisexual adults. Developmental Psychology, 47, 1658-1673. https://doi.org/10.1037/a0025508

Cathey, A. J., \& Wetterneck, C. T. (2013). Stigma and disclosure of intrusive thoughts about sexual themes. Journal of Obsessive-Compulsive and Related Disorders, 2, 439-443. https://doi.org/10.1016/j. jocrd.2013.09.001

Chasson, G. S., Guy, A. A., Bates, S., \& Corrigan, P. W. (2018). They aren't like me, they are bad, and they are to blame: A theoretically-informed study of stigma of hoarding disorder and obsessive-compulsive disorder. Journal of OCD and Related Disorders, 16, 56-65. https://doi.org/10.1016/j.jocrd.2017.12.006

Diamond, L. M. (2003). What does sexual orientation orient? A biobehavioral model distinguishing romantic love and sexual desire. Psychological Review, 110, 173-192. https://doi.org/10.1037/0033-295X.110.1.173 
This article has undergone peer review, been accepted for publication, and posted online before the distribution of the complete issue. It has not yet undergone copy editing and may contain statements and information that have errors. The final version will be available upon completion. Visit www.springerpub.com for more info.

180 Luxon et al.

Diamond, L. M. (2009). Sexual fluidity: Understanding women's love and desire. Harvard University Press.

Floyd, F. J., \& Stein, T. S. (2002). Sexual orientation identity formation among gay, lesbian and bisexual youths: Multiple patterns of milestone experiences. Journal of Research on Adolescence, 12, 167-191. https://doi.org/10.1111/1532-7795.00030

Friedman, M. S., Marshal, M. P., Guadamuz, T. E., Wei, C., Wong, C. F., Saewyc, E. M., \& Stall, R. (2011). A meta-analysis of disparities in childhood sexual abuse, parental physical abuse, and peer victimization among sexual minority and sexual nonminority individuals. American Journal of Public Health, 101, 1481-1494. https://doi.org/10.2105/AJPH.2009.190009

Galupo, M. P. (2018). Plurisexual identity labels and the marking of bisexual desire. In D. Joy Swan \& S. Habbibi (Eds.), Bisexuality: Research, theoretical applications, and recommendations for the invisible sexuality. Springer Publishing Company.

Galupo, M. P., \& Bauerband, L. A. (2016). Sexual orientation reflection and rumination scale: Development and psychometric evaluation. Stigma and Health, 1, 44-58. https://doi.org/10.1037/sah0000017

Galupo, M. P., Davis, K. S., Grynkiewicz, A. L., \& Mitchell, R. C. (2014). Conceptualization of sexual orientation identity among sexual minorities: Patterns across sexual and gender identity. Journal of Bisexuality, 14, 433-456. https://doi.org/10.1080/15299716.2014.933466

Galupo, M. P., Mitchell, R. C., \& Davis, K. S. (2015). Sexual minority self-identification: Multiple identities and complexity. Psychology of Sexual Orientation and Gender Diversity, 2, 355-364. https://doi. org/10.1037/sgd0000131

Galupo, M. P., Mitchell, R. C., Grynkiewicz, A. L., \& Davis, K. S. (2014). Sexual minority reflections on the Kinsey scale and the Klein sexual orientation grid: Conceptualization and measurement. Journal of Bisexuality, 14(3-4), 404-432.

Galupo, M. P., Ramirez, J. L., \& Pulice-Farrow, L. (2017). “Regardless of their gender”: Descriptions of sexual identity among bisexual, pansexual, and queer identified individuals. Journal of Bisexuality, 17, 108-124. https://doi.org/10.1080/15299716.2016.1228491

Glazier, K., Calixte, R. M., \& Rothschild, R. (2013). High rates of OCD symptom misidentification by mental health professionals. Annals of Clinical Psychiatry, 25, 201-209.

Goldberg, R. L. (1984). Heterosexual panic. The American Journal of Psychoanalysis, 44, 209-211. https:// doi.org/10.1007/BF01248300

Gonzalez, K. A., Ramirez, J., \& Galupo, M. P. (2018). Increase in LGBTQ minority stress following the 2016 U. S. Presidential election. Journal of GLBT Family Studies, 14, 130-151. https://doi.org/10.1080/ 1550428X.2017.1420849

Harper, A., Finnerty, P., Martinez, M., Brace, A., Crethar, H. C., Loos, B., Harper, B., Graham, S., Singh, A., Kocet, M., Travis, L., Lambert, S., Burnes, T., Dickey, L. M., Hammer, T. R., \& ALGBTIC LGBQQIA Competencies Taskforce. (2013). Association for lesbian, gay, bisexual, and transgender issues in counseling competencies for counseling with lesbian, gay, bisexual, queer, questioning, intersex, and ally individuals. Journal of LGBT Issues in Counseling, 7, 2-43. https://doi.org/10.1080/15538605. 2013.755444

Hatzenbuehler, M. L. (2009). How does sexual minority stigma 'get under the skin'? A psychological mediation framework. Psychological Bulletin, 135, 707-730. https://doi.org/10.1037/a0016441

Hatzenbuehler, M. L., McLaughlin, K. A., \& Nolen-Hoeksema, S. (2008). Emotion regulation and internalizing symptoms in a longitudinal study of sexual minority and heterosexual adolescents. Journal of Child Psychology and Psychiatry, 49, 1270-1278. https://doi.org/10.1111/j.1469-7610.2008.01924.x

Katz-Wise, S. L. (2015). Sexual fluidity in young adult women and men: Associations with sexual orientation and sexual identity development. Psychology \& Sexuality, 6, 189-208. https://doi.org/10.1080/ 19419899.2013.876445

Katz-Wise, S. L., \& Hyde, J. S. (2012). Victimization experiences of lesbian, gay, and bisexual individuals: A meta-analysis. Journal of Sex Research, 49, 142-167. https://doi.org/10.1080/00224499.2011.637247

Klein, F., Sepekoff, B., \& Wolf, T. J. (1985). Sexual orientation: A multi-variable dynamic process. Journal of Homosexuality, 11(1-2), 35-49. 
This article has undergone peer review, been accepted for publication, and posted online before the distribution of the complete issue. It has not yet undergone copy editing and may contain statements and information that have errors. The final version will be available upon completion. Visit www.springerpub.com for more info.

Repetitive Thoughts About Sexual Orientation $\quad 181$

Laumann, E. O., Gagnon, J. H., Michael, R. T., \& Michaels, S. (1994). The social organization of sexuality: Sexual practices in the United States. University of Chicago Press.

McCarn, S. R., \& Fassinger, R. E. (1996). Revisioning sexual minority identity formation: A new model of lesbian identity and its implications for counseling and research. The Counseling Psychologist, 24, 508-534. https://doi.org/10.1177/0011000096243011

Meyer, I. H. (2003). Prejudice, social stress, and mental health in lesbian, gay, and bisexual populations: Conceptual issues and research evidence. Psychological Bulletin, 129, 674-697. https://doi. org/10.1037/0033-2909.129.5.674

Meyer, I. H. (2013). Prejudice, social stress, and mental health in lesbian, gay, and bisexual populations: Conceptual issues and research evidence. Psychology of Sexual Orientation and Gender Diversity, 1, 3-26. https://doi.org/10.1037/2329-0382.1.S.3

Morgan, E. M., \& Thompson, E. M. (2011). Process of sexual orientation questioning among heterosexual women. Journal of Sex Research, 48, 16-28. https://doi.org/10.1080/00224490903370594

Morgan, E. M., Steiner, M. G., \& Thompson, E. M. (2010). Process of sexual orientation questioning among heterosexual men. Men and Masculinities, 12, 425-443. https://doi.org/10.1177/1097/184X08322630

Mowrer, O. H. (1947). On the dual nature of learning: A reinterpretation of "conditioning" and "problem solving.". Harvard Educational Review, 17, 102-148.

Nolen-Hoeksema, S., Wisco, B. E., \& Lyubomirsky, S. (2008). Rethinking rumination. Perspectives on Psychological Science, 3, 400-424. http://www.jstor.org/stable/40212262

Pega, F., Gray, A., Veale, J. F., Binson, D., \& Sell, R. L. (2013). Toward global comparability of sexual orientation data in official statistics: A conceptual framework of sexual orientation for health data collection in New Zealand's official statistics system. Journal of Environmental and Public Health, 2013, 473451, 8. https://doi.org/10.1155/2013/473451

Pinto, A., Eisen, J. L., Mancebo, M. C., Greenberg, B. D., Stout, R. L., \& Rasmussen, S. A. (2007). Taboo thoughts and doubt/checking: A refinement of the factor structure for obsessive-compulsive disorder symptoms. Psychiatry Research, 151, 255-258. https://doi.org/10.1016/j.psychres.2006.09.005

Plöderl, M., \& Tremblay, P. (2015). Mental health of sexual minorities. A systematic review. International Review of Psychiatry, 27, 367-385. https://doi.org/10.3109/09540261.2015.1083949

Rachman, S. (1997). A cognitive theory of obsessions. Behaviour Research and Therapy, 35, 793-802. https:// doi.org/10.1016/S0005-7967(97)00040-5

Rosario, M., \& Schrimshaw, E. W. (2014). Theories and etiologies of sexual orientation. In D. L. Tolman, L. M. Diamond, J. A. Bauermeister, W. H. George, J. G. Pfaus, \& L. M. Ward (Eds.), APA handbook of sexuality and psychology, Vol. 1: Person-based approaches (pp. 555-596). American Psychological Association.

Ruscio, A. M., Stein, D. J., Chiu, W. T., \& Kessler, R. C. (2010). The epidemiology of obsessive-compulsive disorder in the National Comorbidity Survey Replication. Molecular Psychiatry, 15, 53-63. https://doi. org/10.1038/mp.2008.94

Russell, G. M., \& Richards, J. A. (2003). Stressor and resilience factors for lesbians, gay men, and bisexuals confronting antigay politics. American Journal of Community Psychology, 31(3-4), 313-328.

Salkovskis, P. M. (1985). Obsessional-compulsive problems: A cognitive-behavioural analysis. Behaviour Research and Therapy, 23, 571-583. https://doi.org/10.1016/0005-7967(85)90105-6

Steinberg, D. S., \& Wetterneck, C. T. (2017). OCD taboo thoughts and stigmatizing attitudes in clinicians. Community Mental Health Journal, 53, 275-280. https://doi.org/10.1007/s10597-016-0055-x

Trapnell, P. D., \& Campbell, J. D. (1999). Private self-consciousness and the five-factor model of personality: Distinguishing rumination from reflection. Journal of Personality and Social Psychology, 76, 284-304. https://doi.org/10.1037/0022-3514.76.2.284

Treynor, W., Gonzalez, R., \& Nolen-Hoeksema, S. (2003). Rumination reconsidered: A psychometric analysis. Cognitive Therapy and Research, 27, 247-259. https://doi.org/10.1023/A:1023910315561

Turner, S. M., Beidel, D. C., \& Stanley, M. A. (1992). Are obsessional thoughts and worry different cognitive phenomenon? Clinical Psychology Review, 12, 257-270. https://doi.org/10.1016/0272-7358(92)90117-Q 
van Anders, S. M. (2015). Beyond sexual orientation: Integrating gender/sex and diverse sexualities via sexual configurations theory. Archives of Sexual Behavior, 44, 1177-1213. https://doi.org/10.1007/ s10508-015-0490-8

Wahl, K., Ertle, A., Bohne, A., Zurowski, B., \& Kordon, A. (2011). Relations between a ruminative thinking style and obsessive-compulsive symptoms in non-clinical samples. Anxiety, Stress, \& Coping, 24, 217-225. https://doi.org/10.1080/10615806.2010.482985

Ward, J. (2008). Dude-sex: White masculinities and 'authentic' heterosexuality among dudes who have sex with dudes. Sexualities, 11, 414-434. https://doi.org/10.1177/1363460708091742

Wetterneck, C. T., Smith, A. H., Burgess, A. J., \& Hart, J. M. (2011). Distress from sexual thoughts: Do religiosity, emotions, and thought appraisal matter? Journal of Cognitive Psychotherapy, 25, 189-202. https:// doi.org/10.1891/0889-8391.25.3.189

Williams, M. (2008). Homosexuality anxiety: A misunderstood form of OCD. In L. V. Sebeki (Ed.), LeadingEdge Health Education Issues (pp. 195-205). Nova Science Publishers, Inc.

Williams, M. T., Ching, T. H. W., Tellawi, G., Siev, J., Dowell, J., Schlaudt, V., Slimowicz, J., \& Wetterneck, C. T. (2018). Assessing sexual orientation symptoms in obsessive-compulsive disorder: Development and validation of the sexual orientation obsessions and reactions test (SORT). Behavior Therapy, 49(5), 715-729. https://doi.org/10.1016/j.beth.2017.12.005

Williams, M. T., Crozier, M., \& Powers, M. B. (2011). Treatment of sexual orientation obsessions in obsessive-compulsive disorder using exposure and ritual prevention. Clinical Case Studies, 10, 53-66. https:// doi.org/10.1177/1534650110393732

Williams, M. T., \& Farris, S. G. (2011). Sexual orientation obsessions in obsessive-compulsive disorder: Prevalence and correlates. Psychiatry Research, 187, 156-159. https://doi.org/10.1016/ j.psychres.2010.10.019

Williams, M. T., Mugno, B., Franklin, M. E., \& Faber, S. (2013). Symptom dimensions in obsessivecompulsive disorder: Phenomenology and treatment with exposure and ritual prevention. Psychopathology, 46, 365-376. https://doi.org/10.1159/000348582

Williams, M. T., Slimowicz, J., Tellawi, G., \& Wetterneck, C. (2014). Sexual orientation symptoms in obsessive compulsive disorder: Assessment and treatment with cognitive behavioral therapy. Directions in Psychiatry, 34, 37-50.

Williams, M. T., \& Wetterneck, C. T. (2019). In M. T. Williams \& C. T. Wetterneck (Eds.), Sexual obsessions in obsessive-compulsive disorder: A step-by-step, definitive guide to understanding, diagnosis, and treatment. Oxford University Press. https://doi.org/10.1093/med-psych/9780190624798.001.0001

Williams, M. T., Wetterneck, C., Tellawi, G., \& Duque, G. (2014). Domains of distress among people with sexual orientation obsessions. Archives of Sexual Behavior, 44, 783-789. https://doi.org/10.1007/ s10508-014-0421-0

Wolff, M., Wells, B., Ventura-DiPersia, C., Renson, A., \& Grov, C. (2017). Measuring sexual orientation: A review and critique of U.S. data collection efforts and implications for health policy. Journal of Sex Research, 54, 507-531. https://doi.org/10.1080/00224499.2016.1255

Disclosure. The authors have no relevant financial interest or affiliations with any commercial interests related to the subjects discussed within this article.

Funding. The author(s) received no specific grant or financial support for the research, authorship, and/or publication of this article.

Correspondence regarding this article should be directed to Alexandria M. Luxon, MA, Illinois Institute of Technology, Department of Psychology, 201 Tech Central, 3424 South State Street, Chicago, IL 60616. E-mail: aluxon@hawk.iit.edu 